\title{
Tropenmedizin im Zeitalter hoher globaler Mobilität
}

\author{
Schistosomiasis bei einer Schulklasse nach Ruandareise \\ Tropical Medicine in an Age of High Global Mobility
}

\author{
Schistosomiasis in a School Class after Travel to Rwanda
}

Autoren

Christina Mickan, Thomas Junghanss, Marija Stojkovic

\section{(c) (1) $\circledast \oplus$}

Institut

Zentrum für Infektiologie, Sektion Klinische Tropenmedizin, Universitätsklinikum Heidelberg

\section{Schlüsselwörter}

Schistosomiasis, Bilharziose, Katayama-Syndrom, Eosinophilie, vernachlässigte Erkrankungen

Key words

schistosomiasis, bilharzia, katayama-syndrome, eosinophilia, neglected tropical diseases (NTDs)

\section{Bibliografie}

DOI https://doi.org/10.1055/a-0852-4783

Online-Publikation: 16.5.2019

Dtsch Med Wochenschr 2019; 144: e109-e113

(C) Georg Thieme Verlag KG, Stuttgart · New York

ISSN 0012-0472

Korrespondenzadresse

Dr. med. Christina Mickan

Zentrum für Infektiologie, Sektion Klinische Tropenmedizin, Universitätsklinikum Heidelberg, INF 324, 69120 Heidelberg christina.mickan@web.de

\section{ZUSAMMENFASSUNG}

Einleitung Die Schistosomiasis gehört zu den häufigsten Parasitosen weltweit und führt unbehandelt abhängig von der Parasitenlast zu intestinalen (Schistosoma mansoni, S. japonicum, S. mekongi) und urogenitalen (S. haematobium) chronischen Krankheitsmanifestationen. Die frühe Phase ist geprägt von einer Immunkomplex-vermittelten akuten Symptomatik.

Methoden Wir untersuchten 12 Schüler eines deutschen Gymnasiums, die im Rahmen eines 14-tägigen Schulausfluges in Ruanda im Kivu-See im Westen des Landes gebadet hatten. Nach Rückkehr nach Deutschland erkrankte einer der Schüler an einer akuten Schistosomiasis (Katayama-Syndrom), was die Untersuchung und Therapie der anderen Schüler zur Folge hatte.

Ergebnisse Die bei der Erstvorstellung erhobenen positiven Antikörperresultate wiesen bei allen Schülern auf eine Schis-
tosoma-Infektion hin. Bei 9 Patienten bestand eine Eosinophilie im Differenzialblutbild, bei 3 Patienten wurden Eier von S. mansoni im Stuhl nachgewiesen. 11 der 12 Schüler waren bei der Vorstellung in unserer Ambulanz asymptomatisch. Alle Patienten erhielten eine ambulante Therapie mit Praziquantel und eine 12-monatige Nachsorge.

Diskussion Fernreisen in tropische Regionen werden immer häufiger, schulische und universitäre Partnerschaften, sowie humanitäre Einsätze führen bereits junge Menschen in Entwicklungsländer. Tropenspezifische Erkrankungen werden in Zukunft aufgrund der hohen globalen Mobilität in nicht-endemischen Ländern zunehmen. Für deren Prävention und Diagnostik ist eine über die Spezialambulanzen hinausgehende Sensibilisierung der Ärzteschaft erforderlich. Die Erkrankung verläuft häufig asymptomatisch, eine Dunkelziffer an nicht erkannten Infektionen kann daher nicht ausgeschlossen werden. Bei dem Verdacht oder der Behandlung einer Schistosomiasis sollte immer ein spezialisiertes Zentrum hinzugezogen werden.

\section{ABSTRACT}

Introduction Schistosomiasis is one of the most common parasitic diseases worldwide. If left untreated, intestinal (Schistosoma mansoni, S. japonicum, S. mekongi) and urogenital (S. haematobium) chronic disease manifestations occur depending on the parasite load. The early phase however is characterized by fever and an immune-complex-mediated illness. Long-distance travel to tropical regions is on the rise, academic partnerships and humanitarian missions take even young people to developing countries.

Methods 12 students from a German secondary school had fresh water exposure during a 14-day school trip to Rwanda in Lake Kivu in the west of the country. After returning to Germany, one of the students fell ill with acute schistosomiasis (Katayama syndrome), which led to examination of the other students. WBC, differential blood count, serology for schistosomal antibodies and ova detection in urine and stool were performed on first presentation and 6 and 12 months after therapy.

Results Positive antibody results indicated infection in all students, eosinophilia was found in 9 patients, ova of S. mansoni were detected in 3 cases. At presentation in our outpatient 
department 11 of the 12 students were asymptomatic. All patients received therapy with praziquantel.

Discussion Tropical diseases will further increase due to high global mobility. For their prevention and diagnosis physicians need to be sensitized beyond subject specific units. We describe an outbreak of schistosomiasis in a school class to sensitize physicians outside endemic areas. Since the disease is often asymptomatic a high number of unrecognized infections and illnesses can be assumed. When suspecting or treating schistosomiasis, a specialized center should always be consulted.

\section{Einleitung}

Die Schistosomiasis, auch Bilharziose genannt, gehört zu den häufigsten Parasitosen der Welt. Laut Schätzungen der WHO betrifft sie über 200 Mio. Menschen in über 74 Ländern [1]. Die Erkrankung kommt vornehmlich auf dem afrikanischen Kontinent vor und ist mit Kontakt zu Süßwasser und unzureichendem Zugang zu sanitären Anlagen assoziiert. Man unterscheidet ein akutes Stadium, das Katayama-Syndrom, vom chronischen Krankheitsstadium, welches langfristig zu einer Schädigung innerer Organe führt.

Unter den 5 Spezies innerhalb der Gattung der Pärchenegel (Schistosoma) ist die Hauptkrankheitslast auf S. mansoni (Erreger der intestinalen Bilharziose) und S. haematobium (Erreger der urogenitalen Bilharziose) zurückzuführen. Süßwasserschnecken (Bulinus spp., Biomphalaria spp.) dienen als Zwischenwirt und setzen das für den Menschen infektiöse Larvenstadium (Zerkarien) frei. Die Zerkarien durchdringen die Haut des Menschen während des Süßwasserkontaktes und reifen während ihrer Wanderung durch den Körper zu adulten Würmern. Je nach Spezies leben diese in Venengeflechten des Darms (S. mansoni) oder des Urogenitaltraktes (S. haematobium). Das Weibchen produziert Eier, die z. T. im Stuhl (S. mansoni) bzw. Urin (S. haematobium) ausgeschieden werden, z. T. verbleiben sie im umliegenden Gewebe und führen hier zu chronischen Entzündungsreaktionen, Fibrose und Organschäden [2 - 4]. Selten kann es zu ektoper Lokalisation der Parasiten kommen, sodass Wurmeier z. B. in das ZNS oder Myelon gelangen können. Dies kann zu schwerwiegenden Komplikationen, z. B. einer Myelitis oder Paraparese, führen [5].

Initial kann die Penetration der Haut durch die Zerkarien zu einem juckenden Hautausschlag (sog. Zerkarien-Dermatitis) nach dem Süßwasserkontakt führen. Innerhalb von 2-12 Wochen nach Süßwasserkontakt kann es zu einem akuten hochfieberhaften Krankheitsbild, dem Katayama-Syndrom, kommen. Es entsteht durch die Ablagerung von Antigen-Antikörperkomplexen als Reaktion des Körpers auf die Parasiten-Antigene während der Gewebsphase (Immunkomplexsyndrom). Typischerweise bestehen Fieber, Husten, Myalgien, Arthralgien und eine ausgeprägte Eosinophilie im peripheren Blut [4, 6, 7].

In Deutschland betrifft das Katayama-Syndrom v. a. Reiserückkehrer [8-14]. Spätfolgen betreffen hier hauptsächlich chronisch exponierte Patienten, i. d.R. mit Migrationshintergrund. Dazu gehören z. B. eine portale Leberfibrose, portale Hypertension mit Ausbildung von Umgehungskreisläufen und Komplikationen wie Ösophagusvarizenblutungen oder die Ausbildung eines Blasenzellkarzinoms bei urogenitaler Bilharziose.
Der Direktnachweis der Parasiten-Eier mittels Urin-/Stuhlmikroskopie kann erst nach Reifen der Parasiten zu adulten Würmern, also nach einer Präpatenzzeit von mind. 12 Wochen, gelingen. Mittels serologischer Untersuchungen kann die Infektion indirekt bereits während der Präpatenzzeit nachgewiesen werden, meist besteht eine Eosinophilie im Differenzialblutbild $[2-4,6,15,16]$.

Die Therapie der Schistosomiasis erfolgt i. d. R. nach Ablauf der Präpatenzeit von 12 Wochen gewichtsadaptiert mit 3 Dosen Praziquantel. Praziquantel hat nur eine eingeschränkte Wirksamkeit gegen juvenile Schistosomen, daher wird die frühe antiparasitäre Therapie beim Katayama-Syndrom kontrovers diskutiert. Die Therapie des Katayama-Syndroms ist wenig standardisiert. In der Literatur werden rein symptomatische Behandlungen mit Kortikosteroiden, Kombinationen von Praziquantel mit Steroiden sowie Praziquantel als Monotherapie empfohlen. Ein biologisch plausibles Argument für die Therapie in der akuten Krankheitsphase ist die potenzielle Reduktion der „Ei-Last“ und somit die Verringerung der Wahrscheinlichkeit einer ektopen Eiablage [16, 17].

Durch die Zunahme touristischer Reisen sowie Ausbildungsund beruflicher Aufenthalte in (sub-) tropischen Gebieten werden auch mehr Infizierte und Erkrankte nach Reiserückkehr verzeichnet. Laut Angaben der Welt-Tourismus-Organisation stieg die Zahl internationaler touristischer Reisen in den letzten 8 Jahren stetig an. 2017 lag sie in Deutschland bei 1323 Millionen [18]. Eine Untersuchung des European Network for Tropical Medicine and Travel Health zählte 1465 Fälle importierter Schistosomiasis zwischen 1997 und 2010. Hierbei waren 42,4\% der Patienten bei Diagnose der Infektion asymptomatisch [19].

\section{Patienten}

Wir untersuchten eine Gruppe deutscher Schüler $(n=12)$, davon 7 weiblich und 5 männlich, im Alter von 15-18 Jahren, welche im Rahmen eines 14-tägigen Ruanda-Aufenthaltes in einem Süßwassersee (Kivu-See) im Westen des Landes gebadet hatten. Keiner der Patienten hatte sich zuvor jemals in einem Schistosomiasis-Endemiegebiet aufgehalten.

\section{Methoden}

Erhoben wurden bei der Erstvorstellung, neben epidemiologischen und klinischen Daten, die Dauer des Süßwasserkontaktes, Symptome und Befunde einer Zerkarien-Dermatitis oder eines Katayama-Syndroms, sowie Blutbild, Differenzialblutbild, Schistosoma-Serologie und Direktnachweis mittels Sammelurin und 
Stuhluntersuchung. Für den Antikörpernachweis verwendeten wir folgende hausinterne Tests: Indirekter Immunfluoreszenstest (IIFT) mit S.-mansoni-Männchen als Antigen und den Enzymlinked-Immunosorbent-Assay (ELISA) mit Schistosoma-Ei-Antigen. Diese Tests sind akkreditiert nach DIN EN ISO 15 189. Für den IIFT gelten Werte ab einem Titer von $>1: 20$ als positiv, der ELISA gilt ab Werten $>0,3$ als positiv, Werte zwischen 0,2-0,3 gelten als grenzwertig.

Für den Direktnachweis erfolgte die Mikroskopie auf Parasiteneier des Sediments von Stuhl bzw. von Sammelurinuntersuchungen zwischen 10.00 und 14.00 Uhr.

Alle Patienten erhielten eine Abdomensonografie hinsichtlich Organmanifestationen einer Schistosomiasis gemäß des standardisierten WHO-Ultraschall-Protokolls.

Die Nachsorge umfasste regelmäßige klinische Untersuchungen und Kontrollen des Differenzialblutbildes sowie eine Abschlussuntersuchung 12 Monate nach PZQ-Therapie mit erneuter parasitologischer Urin- und Stuhlmikroskopie auf SchistosomenEier.

\section{Ergebnisse}

Einer der Schüler stellte sich nach Rückkehr aufgrund rezidivierender Fieberepisoden in unserer Ambulanz vor. Zu diesem Zeitpunkt lag die Exposition 10 Wochen zurück. Bei diesem Patienten konnten wir anhand der Klinik, einer Hypereosinophilie und hochpositiver Antikörpertiter ein Katayama-Syndrom diagnostizieren. Daraufhin erfolgte die Untersuchung der anderen Schüler. Die serologischen Resultate bei Erstvorstellung wiesen bei allen auf einen Kontakt zu Erregern der Schistosomiasis hin (12/12). Anamnestisch waren neben dem Patienten mit KatayamaSyndrom bei 6 weiteren Patienten selbstlimitierende, fieberhafte Krankheitsbilder im Intervall zwischen Exposition und Erstvorstellung in unserer Ambulanz aufgetreten. Hierbei handelte es sich um Fieber ohne Fokus $(n=1)$, Fieber und Erkältungssymptome $(n=2)$ (Rhinorrhoe, Halsschmerzen) und Fieber mit trockenem Husten und Arthralgien ( $n=3)$.

Symptome einer Zerkarien-Dermatitis waren keinem der Patienten erinnerlich. Eine Eosinophilie (eosinophile Granulozyten abs. $>500 \times 10^{3} / \mu$ l) war zum Zeitpunkt der Erstvorstellung bei 9 der insgesamt 12 Patienten feststellbar, davon 4 Fälle mit Hypereosinophilie (eosinophile Granulozyten abs. $>1500 \times 10^{3} / \mu \mathrm{l}$ ). Bei 3 Patienten konnten Eier von S. mansoni im Stuhl nachgewiesen werden. Abdomensonografisch zeigten sich bei keinem Patienten Auffälligkeiten.

Alle Patienten erhielten eine Therapie mit Praziquantel $40 \mathrm{mg} / \mathrm{kg} / \mathrm{d}$ p. o. als Einzeldosis an je 3 Tagen. Die erste Gabe erfolgte unter klinischer Überwachung in unserer Ambulanz. Bei guter Verträglichkeit erfolgten die weiteren Einnahmen ambulant jeweils abends vor dem Schlafengehen. Bei Vorliegen einer Eosinophilie von > $1000 \times 10^{3} / \mu$ l eosinophilen Granulozyten absolut erfolgte eine begleitende Medikation mit Prednisolon $40 \mathrm{mg}$ für 5 Tage.

Bei der Abschlussuntersuchung 12 Monate nach Therapie fand sich bei keinem Patienten eine persistierende Eosinophilie. Alle untersuchten Patienten hatten nach Abschluss von 12 Monaten noch nachweisbare Antikörpertiter.

Bei dem Patienten mit Katayama-Syndrom traten erstmalig 16 Tage nach Süßwasserkontakt Fieber und Halsschmerzen auf. Unter Annahme einer Streptokokken-Angina erfolgte eine antibiotische Therapie, worunter die Beschwerden abklangen. Die nächste Fieberepisode mit Abgeschlagenheit und Nackenschmerzen ereignete sich 6 Wochen nach Süßwasserkontakt und war selbstlimitierend nach 5 Tagen. Bei der dritten Fieberepisode von 4 Tagen Dauer mit Gelenkschmerzen, trockenem Husten und erneuten Nackenschmerzen erfolgte eine Blutentnahme, bei der eine Hypereosinophilie von $22 \%$ bei $13000 \times 10^{3} / \mu$ l Leukozyten (entsprechend $2860 \times 10^{3} / \mu$ l eosinophilen Granulozyten absolut) auffiel. Daraufhin erfolgte die Erstvorstellung in unserer Ambulanz, insgesamt 10 Wochen nach Süßwasserkontakt. Laborchemisch bestand weiterhin eine Hypereosinophilie von $37 \%$ bei $13000 \times 10^{3} / \mu$ l Leukozyten (entsprechend $4810 \times 10^{3} / \mu$ l eosinophilen Granulozyten absolut). Entzündungsparameter, Transaminasen und Nieren-Retentionsparameter lagen im Normbereich. Die Serologie ergab hochpositive Schistosoma-Antikörpertiter, der Direktnachweis in Sammelurin und Stuhluntersuchung war negativ.

Der Patient erhielt eine Therapie mit Praziquantel $40 \mathrm{mg} / \mathrm{kgKG} / \mathrm{d}$ an insgesamt 3 Tagen. 2 Dosen wurden im akuten Katayama-Syndrom eingenommen, hierbei erfolgte eine Begleittherapie mit Prednisolon 40 mg/d p. o. mit Beginn 4 Tage vor der antiparasitären Therapie. Anschließend wurde Prednisolon stufenweise über 5 Tage ausgeschlichen. Hier zeigte sich ein starker Anstieg der eosinophilen Granulozyten trotz begleitender Glukokortikoid-Therapie 2 Tage nach der ersten Praziquantel-Einnahme ( $\mathbf{A}$ bb. 1). Im weiteren Verlauf war die Eosinophilie rückläufig, erneute Urin- und Stuhluntersuchung auf Schistosomen-Eier waren nach Ablauf der Präpatenzzeit weiterhin negativ. 16 Wochen nach Süßwasserkontakt erfolgte eine erneute Praziquanteltherapie mit $40 \mathrm{mg} / \mathrm{kg} \mathrm{KG}$ als Einmaldosis, ohne begleitende Steroidgabe. Die Therapie wurde vom Patienten gut vertragen. Bei der Abschlussuntersuchung 12 Monate nach Therapie war der Patient beschwerdefrei, laborchemisch ergaben sich keinerlei Auffälligkeiten (eosinophile Granulozyten normwertig).

\section{Diskussion}

Der von uns beschriebene Schistosomiasis-Ausbruch in einer deutschen Schülergruppe zeigt, dass tropenspezifische und parasitäre Erkrankungen für die ärztliche Praxis zunehmend bedeutsam sind. Während die chronische Schistosomiasis ein relevantes Problem für langjährig-exponierte Menschen darstellt, betrifft die akute Form der Schistosomiasis Personen, die erstmalig in Kontakt mit den Krankheitserregern kommen. Die Infektionsrate ist auch nach nur kurzer Exposition hoch. Da Patienten nach einer Infektion oft asymptomatisch bleiben, ist mit einer hohen Dunkelziffer an Infizierten zu rechnen, welche nicht im Gesundheitssystem vorstellig werden. 

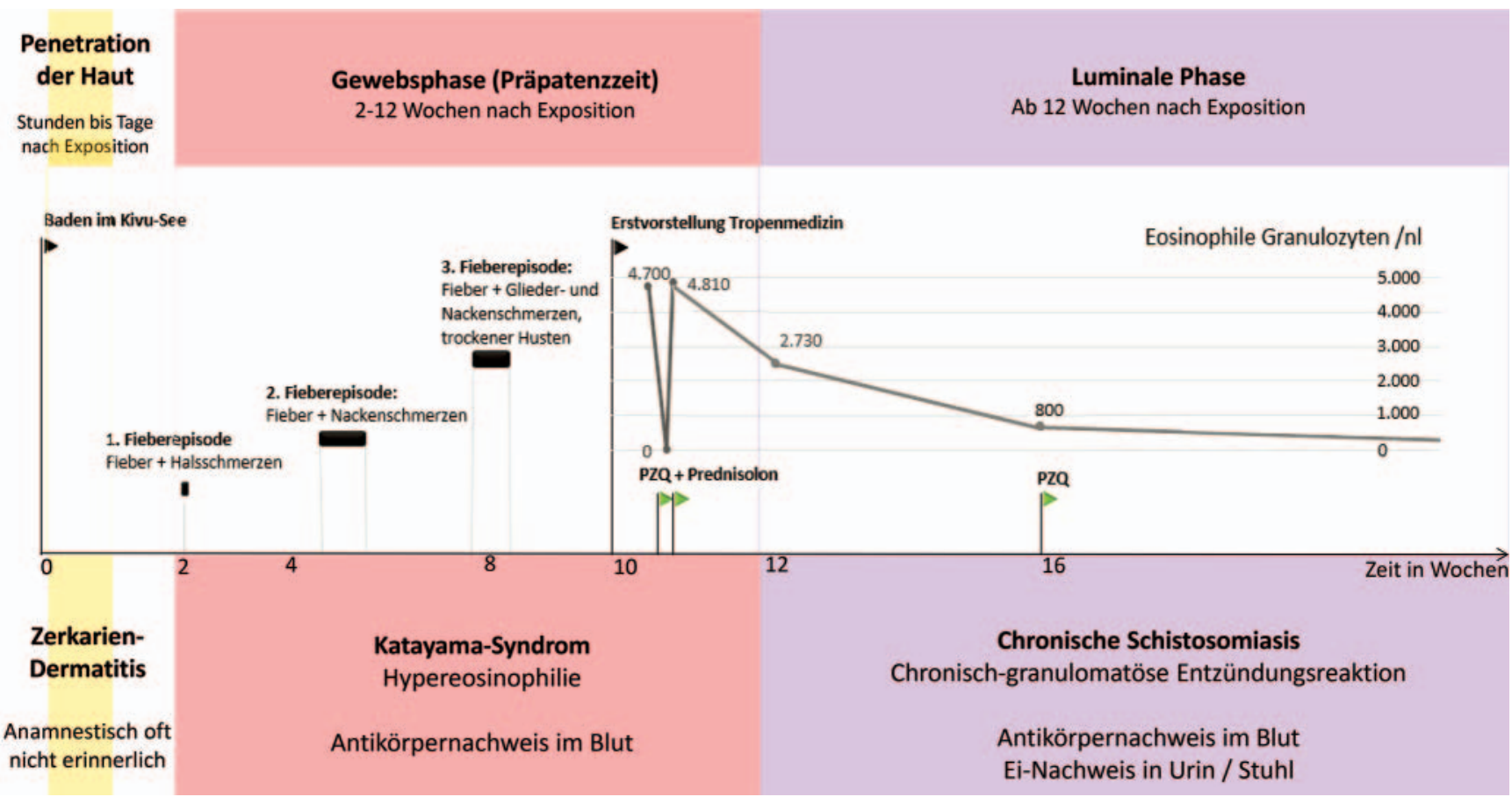

- Abb. 1 Krankheitsverlauf eines Patienten mit Katayama-Syndrom.

Für die Schistosomiasis gefährdete Gruppen sind:

1. touristische Kurzzeitreisende, wie in unserem Beispiel gezeigt,

2. Langzeitreisende, z. B. im Rahmen eines freiwilligen sozialen Jahres (FSJ) und

3. chronisch exponierte Patienten, i. d. R. mit Migrationshintergrund.

1) Touristen Unsere Untersuchung zeigt, dass eine Infektion durch S. mansoni bereits durch äußerst kurzen Süßwasserkontakt möglich ist: Eine unserer Patientinnen gab an, lediglich 2-mal 5 min Wasserkontakt gehabt zu haben. Bei dieser Patientin war neben einer hochpositiven Serologie eine Hypereosinophilie von $17 \%$ bei $12,1 \times 10^{3} / \mu$ l Leukozyten (entsprechend $2057 \times 10^{3} / \mu \mathrm{l}$ eosinophilen Granulozyten absolut) feststellbar. Zudem bestätigt die Untersuchung den Nutzen des Screenings asymptomatischer Reiserückkehrer, welche Süßwasserkontakt im Endemiegebiet hatten: Bei der Erstvorstellung in unserer Ambulanz waren 11 der 12 untersuchten Schüler asymptomatisch. Das Problem wurde der Schulleitung mehrfach eindringlich kommuniziert und die Untersuchung aller anderen möglicherweise Infizierten dringend angeraten.

2) Langzeitreisende Mehrmonatige Auslandsaufenthalte in Entwicklungsländern, z.B im Rahmen eines FSJ, sind insbesondere bei der jüngeren Bevölkerung beliebt. Durch die Teilnahme am täglichen Leben vor Ort ist diese Patientengruppe besonders gefährdet. Das Baden in einer angeblich „Schistosomiasis-freien“ Zone oder angebotene Behandlungen vor Ort führen dazu, dass Patienten sich in falscher Sicherheit wiegen. Die von uns unter- suchten Patienten hatten von einer scheinbar unbewohnten Insel aus gebadet, um dem Risiko einer Infektion zu entgehen. Was die Schüler nicht beachtet hatten war, dass die Insel von Fischern als Ort für die Mittagspause genutzt wurde, sodass die Insel zwar unbewohnt, jedoch nicht unbesucht ist.

3) Patienten mit Migrationshintergrund Diese Patienten sind vom chronischen Stadium der Schistosomiasis betroffen, welches häufig über lange Zeit symptomarm ist. Daher empfehlen wir, dass alle Patienten, die aus einem Endemiegebiet stammen, auf Schistosomiasis gescreent werden. Bei diesen Patienten ist zu beachten, dass im Gegensatz zu touristisch oder Langzeitreisenden serologische Untersuchungen alleine nicht ausreichen, um eine Erkrankung auszuschließen. Hier muss immer auch ein Direktnachweis in Stuhl bzw. Urin geführt werden. Die initiale Abklärung sollte ein Differenzialblutbild, Schistosoma-Antikörper und Stuhluntersuchungen auf Eier von S. mansoni umfassen. Die Mikroskopie des Sammelurins auf Eier von S. haematobium ist i. d. R. nur in Spezialambulanzen mit parasitologischem Labor möglich.

Tropenmedizinische Krankheitsbilder bedürfen einer höheren Aufmerksamkeit in der täglichen ärztlichen Praxis. Die Schistosomiasis gehört zu den häufigsten Parasitosen weltweit und muss bei Reiserückkehrern und Patienten mit Migrationshintergrund beachtet werden. Dies gilt insbesondere im Hinblick auf die zunehmende Reiseaktivität junger Menschen. Da die Erkrankung häufig asymptomatisch verläuft, ist von einer Dunkelziffer nicht erkannter Infektionen bzw. Erkrankungen auszugehen. 


\section{KERNAUSSAGEN}

- Die Schistosomiasis gehört zu den häufigsten Parasitosen weltweit und nimmt aufgrund der hohen globalen Mobilität zu. Da die Infektion häufig asymptomatisch verläuft, ist mit einer hohen Dunkelziffer zu rechnen.

- Ärzte müssen für die Erkrankung sensibilisiert werden, Reisende sollten vor Aufenthalten in Endemiegebieten auf das Risiko einer Infektion und die Präventionsmöglichkeiten hingewiesen werden.

- Langzeitreisende sind aufgrund der Teilnahme am täglichen Leben besonders gefährdet. Vor Ort angebotene Behandlungen können abhängig vom Therapiezeitpunkt ineffektiv sein und zu einer Verschlechterung des klinischen Zustandes führen.

- Bei unklarem Fieber oder Eosinophilie nach Reisen in Endemiegebiete sollte immer auch eine Schistosomiasis differenzialdiagnostisch abgeklärt werden. Auch bei asymptomatischen Reiserückkehrern, die Süßwasserkontakt im Endemiegebiet hatten, muss eine Diagnostik durchgeführt werden.

- Wir empfehlen, Patienten, die aus Endemiegebieten stammen, auf eine Schistosomiasis zu screenen. Die Untersuchung sollte ein Differenzialblutbild, SchistosomaAntikörper sowie die parasitologische Untersuchung von Stuhl und Sammelurin beinhalten.

- Bei Verdacht auf Vorliegen einer Schistosomiasis sollte immer ein tropenmedizinisches Zentrum hinzugezogen werden.

\section{WEITERFÜHRENDE LINKS:}

- Karte zum weltweiten Vorkommen der Schistosomiasis: http://www.who.int/schistosomiasis/Schistosomiasis_ $2012-01$. png?ua $=1$

- Epidemiologie und parasitärer Lebenszyklus: http://www.who.int/schistosomiasis/epidemiology/en/

\section{Interessenkonflikt}

Die Autoren geben an, dass kein Interessenkonflikt besteht.
[1] WHO. Schistosomiasis. Im Internet: https://www.who.int/ schistosomiasis/en/; Stand: 19.04.2019

[2] Bustinduy A. Schistosomiasis. In: Farrar ], Hotez P, Junghanss T, et al, Hrsg Manson's Tropical Diseases. 23. Aufl. Philadelphia: Elsevier Saunders; 2013: $698-725$

[3] Colley DG, Bustinduy AL, Secor WE et al. Human schistosomiasis. Lancet 2014; 383: 2253 -2264. doi:10.1016/S0140-6736(13)61949-2

[4] Maguire JH. Trematodes - Schistosomes and other Flukes. In: Bennett JE, Dolin R, Blaser MJ, Hrsg Mandell, Douglas, and Bennett's Principles and Practice of Infectious Diseases. 8. Aufl. Philadelphia: Churchill Livingstone Elsevier; 2016: 3216-3221

[5] Ross AG, McManus DP, Farrar ] et al. Neuroschistosomiasis. Journal of neurology 2012; 259: 22 - 32. doi:10.1007/s00415-011-6133-7

[6] Doherty JF, Moody AH, Wright SG. Katayama fever: an acute manifestation of schistosomiasis. Bmj 1996; 313: 1071 -1072

[7] Harries AD, Cook GC. Acute schistosomiasis (Katayama fever): clinical deterioration after chemotherapy. The Journal of infection 1987; 14: $159-161$

[8] Alexander CL, Cottom L, Smith K et al. Schistosomiasis in Scottish travellers: public health importance of laboratory testing and the need for enhanced surveillance. Journal of public health 2018; 40: 138-145. doi:10.1093/pubmed/fdx024

[9] Blach O, Rai B, Oates K et al. An outbreak of schistosomiasis in travellers returning from endemic areas: the importance of rigorous tracing in peer groups exposed to risk of infection. Journal of public health 2012; 34: 32 - 36. doi:10.1093/pubmed/fdr099

[10] Corachan M. Schistosomiasis and international travel. Clinical infectious diseases: an official publication of the Infectious Diseases Society of America 2002; 35: 446-450. doi:10.1086/341895

[11] Day JH, Grant AD, Doherty JF et al. Schistosomiasis in travellers returning from sub-Saharan Africa. Bmj 1996; 313: 268-269

[12] Schwartz E, Kozarsky P, Wilson M et al. Schistosome infection among river rafters on Omo River, Ethiopia. Journal of travel medicine 2005; 12 : $3-8$

[13] Visser LG, Polderman AM, Stuiver PC. Outbreak of schistosomiasis among travelers returning from Mali, West Africa. Clinical infectious diseases: an official publication of the Infectious Diseases Society of America 1995; 20: $280-285$

[14] Whitty C], Mabey DC, Armstrong M et al. Presentation and outcome of 1107 cases of schistosomiasis from Africa diagnosed in a non-endemic country. Transactions of the Royal Society of Tropical Medicine and Hygiene 2000; 94: 531 - 534

[15] AWMF. S1-Leitlinie: Diagnostik und Therapie der Schistosomiasis. AWMF-Register Nr 042/005 2013.

[16] Ross AG, Vickers D, Olds GR et al. Katayama syndrome. The Lancet Infectious diseases 2007; 7: 218 -224. doi:10.1016/S14733099(07)70053-1

[17] Bottieau E, Clerinx J, de Vega MR et al. Imported Katayama fever: clinica and biological features at presentation and during treatment. The Journal of infection 2006; 52: 339 -345. doi:10.1016/j.jinf.2005.07.022

[18] World Tourism Organization. UNWTO Annual Report 2017. Madrid: UNWTO. 2018. Statistical Annex

[19] Lingscheid T, Kurth F, Clerinx J et al. Schistosomiasis in European Travelers and Migrants: Analysis of 14 Years TropNet Surveillance Data. The American journal of tropical medicine and hygiene 2017; 97: 567 574. doi:10.4269/ajtmh.17-0034 